\title{
Generalized Hyperbolic Distributions and Brazilian Data*
}

José Fajardo**

Aquiles Farias ${ }^{* * *}$

\begin{abstract}
The aim of this paper is to discuss the use of the Generalized Hyperbolic Distributions to fit Brazilian assets returns. Selected subclasses are compared regarding goodness of fit statistics and distances. Empirical results show that these distributions fit data well. Then we show how to use these distributions in value at risk estimation and derivative price computation.

Keywords: Generalized Hyperbolic Distributions, Derivatives Pricing, Fat Tails, Fast Fourier Transformation.
\end{abstract}

JEL Code: C52, G10.

\footnotetext{
${ }^{*}$ Submitted in September 2002. Revised in July 2004. J. Fajardo thanks CNPq and PRONEX E-26/171.193/2003 for financial support.

${ }^{* *}$ IBMEC Business School. E-mail: pepe@ibmecrj.br

${ }^{* * *}$ University of Brasília. E-mail: aquiles@unb.br
} 


\section{Introduction}

Since Mandelbrot (1963), the behavior of asset returns has been extensively studied. Using low frequency data, he shows that log returns present tails heavier than the Gaussian tails, so he suggested the use of Pareto-stable distributions. Unfortunately these distributions present extremely fat tails, a fact that is refused by empirical evidence. Using high frequency data, other "stylized facts" of real-life returns have been studied, namely: volatility clustering, long range dependence and aggregational Gaussianity. Many econometric models have been suggested to explain part of the behavior of these asset returns, among them we can mention the generalized autoregressive conditionally heteroskedastic model (GARCH). Unfortunately, GARCH can not explain long range dependence. However, long range dependence can be captured with the Fractional Integrated GARCH (FIGARCH) introduced by Baillie et al. (1996). Other models have been suggested to capture other stylized facts, we refer the reader to Rydberg (1997) for a survey of these models.

A usual classification developed in the literature is the one that divides the models into discrete and continuous time models. In this paper we will deal with the latter class. An important class called diffusion models has been largely used by the authors, but the use of a Brownian motion implies the Gaussian distributions of log-returns, a fact that is very well-known not satisfied by the majority of the asset returns.

Recently, a class of distributions called Generalized Hyperbolic Distributions (GHD) has been suggested to fit financial data. The development of these distributions is due to Barndorff-Nielsen (1977). He applied the Hyperbolic subclass to fit grain size of sand subjected to continuous wind blowing. Further, in BarndorffNielsen (1978), the concepts were generalized to the GHD. Since its development, GHD were used in different fields of knowledge such as Physics, Biology ${ }^{1}$ and Agronomy. Eberlein and Keller (1995) were the first to apply these distributions to finance. In their work they use Hyperbolic subclasses to fit German data.

Keller (1997) developed expressions for derivative pricing and Prause (1999) extended Eberlein and Keller (1995) and applied GHD to fit financial data, using German stocks and American indexes. He also prices derivatives, measures Value at Risk (VaR) and extends to the multivariate case of these distributions. In the early 1990s Blæsild and Sørensen (1992) developed a computer program called Hyp which was used to estimate the parameters of Hyperbolic subclass distributions up to three dimensions. Prause (1999) developed a program to estimate the GHD parameters, but the structure of these programs is not freely available.

Some applications to the Brazilian market have been carried out to analyze these stylized facts. Using the Hyp software, Fajardo et al. (2001) analyze the goodness of fit of Hyperbolic distributions (subclass of the GHD). Mendes and

\footnotetext{
${ }^{1}$ For an application to other fields of knowledge we suggest Blæsild and Sørensen (1992).
} 
Duarte Jr. (1999), Issler (1999), Mazuchelli and Migon (1999) and Pereira et al. (1999) use the GARCH model to study Brazilian data.

In this paper we generalize Fajardo et al. (2001) using GHD to fit Brazilian data. We also show how to price derivatives and estimate Value at Risk, which are not addressed by these authors. The main contribution of the paper is to create the parameter estimation algorithm for GHD and to use Fast Fourier Transformation (FFT) in order to obtain the t-fold convolution of the GHD, which is used in derivative pricing. The Fast Fourier Transformation is required because in most cases GHD is not closed under convolution.

The paper is organized as follows. Section 2 presents the Generalized Hyperbolic Distributions and their subclasses. Section 3 presents the data used for this estimation. Section 4 describes the GHD estimation procedures. Section 5 contains the results obtained in GHD estimation. Section 6 presents some statistical tests and distances to evaluate the goodness of fit. Section 7 prices derivatives. Section 8 evaluates the feasibility of VaR measures using GHD. The last sections contain the conclusions and some figures and tables.

\section{Generalized Hyperbolic Distributions}

The probability density function of the one dimensional GHD is defined by:

$$
g h(x ; \alpha, \beta, \delta, \mu, \lambda)=a(\lambda, \alpha, \beta, \delta)\left(\delta^{2}+(x-\mu)^{2}\right)^{\frac{\left(\lambda-\frac{1}{2}\right)}{2}} K(\lambda, \alpha, \beta, \delta, \mu)
$$

where $\mu$ is a location parameter, $\delta$ is a scale factor, compared to Gaussians $\sigma$ in Eberlein (2000), $\alpha$ and $\beta$ determine the distribution shape, $\lambda$ defines the tail fatness Barndorff-Nielsen and Blaesild (1981), therefore the subclasses of GHD, and

$$
K(\lambda, \alpha, \beta, \delta, \mu)=K_{\lambda-\frac{1}{2}}\left(\alpha \sqrt{\delta^{2}+(x-\mu)^{2}}\right) \exp (\beta(x-\mu))
$$

where,

$$
a(\lambda, \alpha, \beta, \delta)=\frac{\left(\alpha^{2}-\beta^{2}\right)^{\frac{\lambda}{2}}}{\sqrt{2 \pi} \alpha^{\left(\lambda-\frac{1}{2}\right)} \delta^{\lambda} K_{\lambda}\left(\delta \sqrt{\alpha^{2}-\beta^{2}}\right)}
$$

is a norming factor to make the curve area equal to 1 and

$$
K_{\lambda}(x)=\frac{1}{2} \int_{0}^{\infty} y^{\lambda-1} \exp \left(-\frac{1}{2} x\left(y+y^{-1}\right)\right) d y
$$

is the modified Bessel function ${ }^{2}$ of third kind with index $\lambda$. The domains of the parameters are:

$$
\begin{gathered}
\mu, \lambda \in \mathbb{R} \\
-\alpha<\beta<\alpha
\end{gathered}
$$

\footnotetext{
${ }^{2}$ For more details about Bessel functions, see Abramowitz and Stegun (1968).
} 


$$
\delta, \alpha>0
$$

Its log-density is hyperbolic while Gaussian distribution log-density is a parabola. For this reason it is called Generalized Hyperbolic.

We can do a reparametrization of the distribution so that the new parameters are scale invariant. The new parameters are defined by:

$$
\begin{array}{ll}
\zeta=\delta \sqrt{\alpha^{2}-\beta^{2}} & \varrho=\frac{\beta}{\alpha} \\
\xi=(1+\zeta)^{-\frac{1}{2}} & \chi=\xi \varrho \\
\bar{\alpha}=\alpha \delta & \bar{\beta}=\beta \delta
\end{array}
$$

The GHD have semi-heavy tails, and they receive this name because their tails are heavier than those in the Gaussian distribution, but they have finite variance, which is observed by the following approximation:

$$
g h(x ; \lambda, \alpha, \beta, \delta) \sim|x|^{\lambda-1} \exp ((\mp \alpha+\beta) x) \text { as } x \rightarrow \pm \infty
$$

Many distributions can be obtained as subclasses or limiting distributions of GHD. We cite as examples the Gaussian, Student's T and Normal Inverse Gaussian distributions. We refer to Barndorff-Nielsen (1978) and Prause (1999) for a detailed description. Using Bessel function simplifications when its index is $\mathbb{N}+\frac{1}{2}$ we can get simpler densities to some subclasses. When $\lambda=1$ we have the Hyperbolic Distribution subclass (H). As shown in (1), the Bessel function appears only in the norming factor, which makes the maximum likelihood estimation easier. The simplified density is given by:

$$
h_{y p}(x ; \alpha, \beta, \delta, \mu)=\frac{\sqrt{\alpha^{2}-\beta^{2}}}{2 \delta \alpha K_{1}\left(\delta \sqrt{\alpha^{2}-\beta^{2}}\right)} \exp \left(-\alpha \sqrt{\delta^{2}+(x-\mu)^{2}}+\beta(x-\mu)\right)
$$

This distribution is not closed under convolution. When we make $\lambda=-0.5$, and using Bessel function properties, we get a distribution called Normal Inverse Gaussian distribution (NIG) whose density is given by:

$$
n i g(x ; \alpha, \beta, \delta, \mu)=\frac{\alpha \delta}{\pi} \exp \left(\delta \sqrt{\alpha^{2}-\beta^{2}}+\beta(x-\mu)\right) \frac{K_{1}\left(\alpha \sqrt{\delta^{2}+(x-\mu)^{2}}\right)}{\sqrt{\delta^{2}+(x-\mu)^{2}}}
$$

This name results from the fact that it can be represented as a mixture of a Generalized Inverse Gaussian with a Normal distribution. More details on these distributions can be found in Rydberg (1997), Keller (1997), Barndorff-Nielsen (1997) and Barndorff-Nielsen (1998). This subclass has the desired property of being closed under convolution, i.e. the convolution of two NIG distributions is another NIG distribution:

$$
\operatorname{nig}^{* t}(x ; \alpha, \beta, \delta, \mu)=\operatorname{nig}(x ; \alpha, \beta, t \delta, t \mu)
$$


The operator $*$ is the convolution operator, thus $n i g^{* t}$ is the density of the distribution of the process at time $t$. This fact makes NIG subclass more adequate to price derivatives, since we need the distribution of the price process at maturity, as we will see in section 7 .

\section{Sample}

The empirical evaluation uses Brazilian assets that have the minimum liquidity requirement. Our sample consists of 14 assets and the Ibovespa index. The assets also represent different economic sectors and public, private and privatized institutions. The data consisted of the daily log-returns, which were calculated using:

$$
R_{i, t}=\ln \left(\frac{P_{i, t}}{P_{i, t-1}}\right)
$$

The price process $\left\{P_{i, t}\right\}$ of each asset $i$ was adjusted according to their rights, such as dividends, splits and groupings. The samples with their respective periods are in Table 1. The chosen starting date was the date at which Brazil implemented its currency stabilization plan (Real plan), which brought some stability to the prices, avoiding daily correction of asset prices. Note that when the starting date of the sample is not $07 / 01 / 1994$ it is because the asset started to be traded only after that date, as these assets resulted from the privatization of Telebras.

Table 1

Sample

\begin{tabular}{l|c|c|c}
\hline Asset & Ticker & Start & End \\
\hline Banco Itaú - PN & Itau4 & $07 / 01 / 1994$ & $12 / 13 / 2001$ \\
Banco do Brasil - PN & Bbas4 & $07 / 01 / 1994$ & $12 / 13 / 2001$ \\
Bradesco - PN & Bbdc4 & $07 / 01 / 1994$ & $12 / 13 / 2001$ \\
Cemig - PN & Cmig4 & $07 / 01 / 1994$ & $12 / 13 / 2001$ \\
Cia Siderúrgica Nacional - ON & Csna3 & $07 / 01 / 1994$ & $12 / 13 / 2001$ \\
Eletrobrás - PNB & Elet6 & $07 / 01 / 1994$ & $12 / 13 / 2001$ \\
Embratel Participações - PN & Ebtp4 & $09 / 21 / 1998$ & $12 / 13 / 2001$ \\
Ibovespa & Ibvsp & $07 / 01 / 1994$ & $12 / 13 / 2001$ \\
Petrobrás - PN & Petr4 & $07 / 01 / 1994$ & $12 / 13 / 2001$ \\
Petrobrás Distribuidora - PN & Brdt4 & $07 / 04 / 1994$ & $12 / 13 / 2001$ \\
Tele Celular Sul - PN & Tcsl4 & $09 / 21 / 1998$ & $12 / 13 / 2001$ \\
Tele Nordeste Celular - PN & Tnep4 & $09 / 21 / 1998$ & $12 / 13 / 2001$ \\
Telemar - PN & Tnlp4 & $09 / 22 / 1998$ & $12 / 13 / 2001$ \\
Telesp - PN & Tlpp4 & $07 / 01 / 1994$ & $12 / 13 / 2001$ \\
Vale do Rio Doce - PNA & Vale5 & $07 / 01 / 1994$ & $12 / 13 / 2001$ \\
\hline
\end{tabular}

\section{Estimation Algorithm}

We developed a MatLab program that uses maximum log-likelihood to estimate the GHD parameters, because Prause (1999) showed that this estimation method is the only one that is non-biased, among a large class of methods used in the 
literature. This method was also used by Blæsild and Sørensen (1992) in the Hyp software, in order to estimate only the multivariate hyperbolic subclass $(\lambda=1)$ parameters. The maximum log-likelihood parameters are those that maximize the following likelihood function:

$$
L=\sum_{i=1}^{n} \log \left(g h\left(x_{i} ; \alpha, \beta, \delta, \mu, \lambda\right)\right)
$$

This estimation consists of a numerical optimization procedure. We use the Downhill Simplex Method which makes no use of derivatives, developed by Nelder and Mead (1965), with some modifications (due to parameter restrictions). It is worth noting that Prause (1999) used a Bracketing Method. The Downhill Simplex Method requires starting values to begin optimization. In this case, we followed Prause (1999) who used a symmetric distribution $(\beta=0)$ with a reasonable kurtosis $(\xi \approx 0.7)$ to equalize the mean and variance of the GHD to those of the empirical probability distribution. This is done because when we use a symmetric distribution and fix the kurtosis, we reduce the computational effort.

In all numerical optimizations we have to define the tolerance of the search, and we decided to use $1 \times 10^{-10}$. This tolerance was applied in absolute ways to the function evaluation and to the parameter sum variation. The numerical maximum likelihood estimation does not have a convergence analytical proof, and it showed empirical convergence even when different starting values were used Prause (1999).

\section{Empirical Results}

In this section we present the estimation results of each subclass.

\subsection{Hyperbolic subclass}

In Table 2 we have the estimated parameters and the log-likelihood (LLH) value. All samples except Cemig have asymmetric distribution estimations since $\beta$ is different from 0 .

In Figure 6, we have the Vale do Rio Doce (Vale5) Hyperbolic subclass estimation compared to the Gaussian estimation and Empirical probability distribution. The figure leads us to visually evaluate the fit of Hyperbolic subclass. The Hyperbolic subclass shows to fit well the leptokurtic behavior of the empirical curve. To see the fitness of the distribution tails we refer to the log-density graph in Figure 6. We can see again that the Hyperbolic distribution is closer to the empirical probability distribution. However, we need a more formal test to verify this closeness, as we will see in section 6 . 
Table 2

Estimated Hyperbolic parameters

\begin{tabular}{l|r|r|r|r|r}
\hline Sample & \multicolumn{1}{|c}{$\alpha$} & \multicolumn{1}{c}{$\beta$} & \multicolumn{1}{c}{$\delta$} & \multicolumn{1}{c}{$\mu$} & \multicolumn{1}{c}{ LLH } \\
\cline { 5 - 6 } Bbas4 & 41.5931 & 3.8960 & 0.0131 & -0.0055 & 3512.08 \\
Bbdc4 & 47.5455 & -0.0006 & 0 & 0 & 3984.49 \\
Brdt4 & 51.7172 & 4.1032 & 0.0119 & -0.0032 & 3925.06 \\
Cmig4 & 43.3673 & 0 & 0.0104 & 0.0004 & 3677.76 \\
Csna3 & 47.4118 & 0.0082 & 0 & 0 & 3976.50 \\
Ebtp4 & 36.7618 & 3.8088 & 0.0197 & -0.0075 & 1409.48 \\
Elet6 & 41.1231 & 1.1727 & 0.0145 & -0.0012 & 3522.58 \\
Ibvsp & 57.6958 & -0.0070 & 0.0096 & 0.0012 & 4165.69 \\
Itau4 & 49.9390 & 1.7495 & 0 & 0 & 4084.89 \\
Petr4 & 45.7651 & 0.7970 & 0.0102 & 0 & 3755.75 \\
Tcs14 & 35.5804 & 0.0004 & 0.0324 & 0.0014 & 1325.28 \\
Tlpp4 & 41.7147 & -0.0051 & 0 & 0 & 3753.45 \\
Tnep4 & 34.9981 & 3.4613 & 0.0310 & -0.0054 & 1314.64 \\
Tnlp4 & 42.7018 & 0.0025 & 0.0207 & 0.0003 & 1501.83 \\
Vale5 & 48.7391 & 2.9889 & 0.0056 & -0.0017 & 3955.42 \\
\hline
\end{tabular}

\subsection{Normal inverse Gaussian subclass}

The Normal Inverse Gaussian Distribution (NIG) $(\lambda=-0.5)$ has been widely used to study German data Prause (1999), and it presents better fit than the Hyperbolic distribution. The estimated parameters and the log-likelihood values for Brazilian assets are in Table 3.

In Figures 8 and 9, we have the density and the log-density graphs of Vale do Rio Doce assets, respectively. We can not see much difference between Hyperbolic and NIG distributions.

\subsection{Generalized hyperbolic distribution}

A GHD is obtained by estimating all five parameters, i.e. the $\lambda$ parameter is also estimated. Prause (1999) was the first to test it empirically on financial data. After that, after this work Raible (2000) pointed out that there is a large diffculty when the parameters $\delta$ and $\mu$ tended simultaneously to zero. The numerical solution to this problem was the use of specific treatments to the case following Hanselman and Littlefield (2001) and Abramowitz and Stegun (1968).

They have never been used with Brazilian data, since Fajardo et al. (2001), only used the Hyperbolic subclass. Table 4 contains the estimated parameters for all samples.

As desired, the GHD estimations had higher log-likelihood values than their subclasses, but in Bradesco and Itaú samples the values are close to the subclass values. The major samples had $\lambda$ between -0.62 and -2.62 , which is similar to the results obtained by Prause (1999). In Figures 1 and 2 we have the comparisons between densities and log-densities, respectively. Then, we can see the better fit of the GHD. 


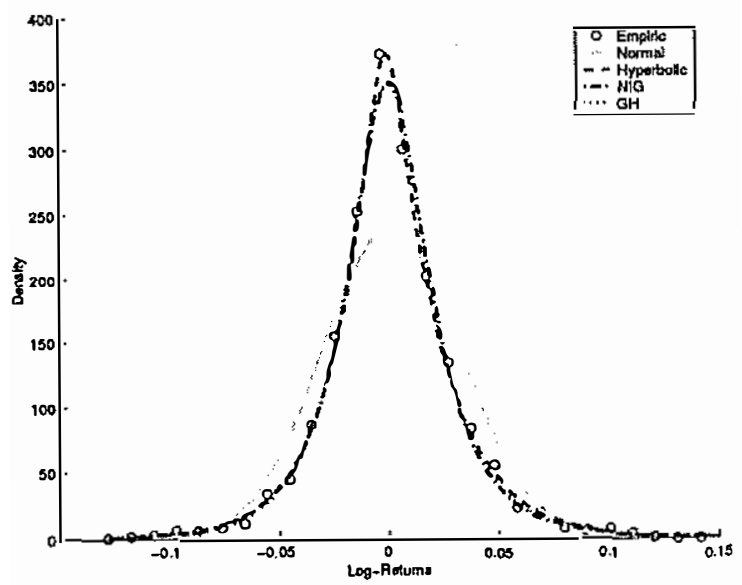

Figure 1

Vale do Rio Doce Probability Distributions: Empirical $x$ Hyperbolic $x$ Normal $x$ NIG $x$ $\mathrm{GH}$

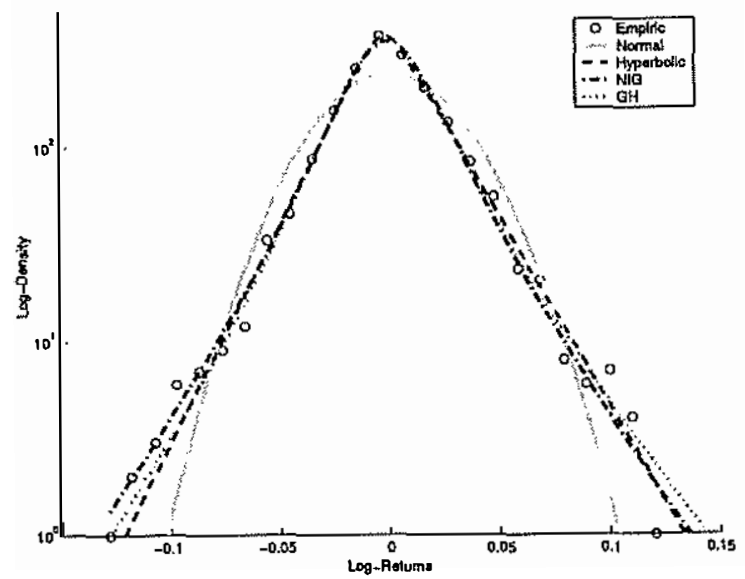

Figure 2

Vale do Rio Doce Log-Probability Distributions: Empirical $x$ Hyperbolic $\mathrm{x}$ Normal $\mathrm{x}$ NIG $\times$ GII 
Table 3

Estimated NIG parameters

\begin{tabular}{c|c|c|c|c|c}
\hline Sample & $\alpha$ & $\beta$ & $\delta$ & $\mu$ & LLH \\
\hline Bbas4 & 26.1863 & 3.3516 & 0.0356 & -0.0049 & 3512.65 \\
Bbdc4 & 25.2340 & 0.0027 & 0.0234 & 0 & 3978.71 \\
Brdt4 & 36.7793 & 3.7894 & 0.0325 & -0.0029 & 3922.97 \\
Cmig4 & 27.0195 & 0.0008 & 0.0327 & 0.0003 & 3681.63 \\
Csna3 & 25.3325 & 2.4753 & 0.0236 & -0.0015 & 3949.86 \\
Ebtp4 & 22.8770 & 3.7054 & 0.0424 & -0.0075 & 1412.84 \\
Elet6 & 23.6626 & 0.0034 & 0.0343 & 0 & 3532.29 \\
Ibvsp & 31.9096 & -0.0035 & 0.0233 & 0.0012 & 4178.17 \\
Itau4 & 30.7352 & 0.0015 & 0.0249 & 0.0008 & 4065.40 \\
Petr4 & 25.3411 & 0.0009 & 0.0285 & 0.0007 & 3764.37 \\
Tcsl4 & 24.5055 & -0.0003 & 0.0556 & 0.0012 & 1327.02 \\
Tlpp4 & 20.3812 & 0.0038 & 0.0249 & 0.0003 & 3763.65 \\
Tnep4 & 21.8374 & 0.0013 & 0.0519 & 0.0012 & 1317.41 \\
Tnlp4 & 26.2133 & 0.0010 & 0.0384 & 0.0004 & 1505.29 \\
Vale5 & 26.6233 & 0.0048 & 0.0249 & 0 & 3956.39 \\
\hline
\end{tabular}

Table 4

Estimated GHD Parameters

\begin{tabular}{c|c|c|c|c|c|c}
\hline Sample & $\alpha$ & $\beta$ & $\delta$ & $\mu$ & $\lambda$ & LLH \\
\hline Bbas4 & 30.7740 & 3.5267 & 0.0295 & -0.0051 & -0.0492 & 3512.73 \\
Bbdc4 & 47.5455 & -0.0006 & 0 & 0 & 1 & 3984.49 \\
Brdt4 & 56.4667 & 3.4417 & 0.0026 & -0.0026 & 1.4012 & 3926.68 \\
Cmig4 & 1.4142 & 0.7491 & 0.0515 & -0.0004 & -2.0600 & 3685.43 \\
Csna3 & 46.1510 & 0.0094 & 0 & 0 & 0.6910 & 3987.52 \\
Ebtp4 & 3.4315 & 3.4316 & 0.0670 & -0.0071 & -2.1773 & 1415.64 \\
Elet6 & 1.4142 & 0.0120 & 0.0524 & 0 & -1.8987 & 3539.06 \\
Ibvsp & 1.7102 & -1.6684 & 0.0357 & 0.0020 & -1.8280 & 4186.31 \\
Itau4 & 49.9390 & 1.7495 & 0 & 0 & 1 & 4084.89 \\
Petr4 & 7.0668 & 0.4848 & 0.0416 & 0.0003 & -1.6241 & 3767.41 \\
Tcs14 & 1.4142 & 0 & 0.0861 & 0.0011 & -2.6210 & 1329.64 \\
Tlpp4 & 6.8768 & 0.4905 & 0.0359 & 0 & -1.3333 & 3766.28 \\
Tnep4 & 2.2126 & 2.2127 & 0.0786 & -0.0028 & -2.2980 & 1323.66 \\
Tnlp4 & 1.4142 & 0.0021 & 0.0590 & 0.0005 & -2.1536 & 1508.22 \\
Vale5 & 25.2540 & 2.6134 & 0.0265 & -0.0015 & -0.6274 & 3958.47 \\
\hline
\end{tabular}

\subsection{Remark on estimation}

Now, although there seems to exist an overparameterization, in many cases the restrictions $\lambda=1$ and $\lambda=-0,5$ can be easily rejected, as shown in the following table: 
Table 5

$P$-Values

\begin{tabular}{l|c|c|c|c|c}
\cline { 5 - 6 } Sample & LLH Hyp & LLH Nig & LLH GH & PV (Hyp) & PV (Nig) \\
\hline Bbas4 & $3.512,08$ & $3.512,65$ & $3.512,73$ & $\mathbf{0 , 2 5 4 2 1 3}$ & $\mathbf{0 , 6 8 9 1 5 7}$ \\
Bbdc4 & $3.984,49$ & $3.978,71$ & $3.984,49$ & $\mathbf{1}$ & 0,000674 \\
Brdt4 & $3.925,06$ & $3.922,97$ & $3.926,68$ & $\mathbf{0 , 0 7 1 8 6 1}$ & 0,00645 \\
Cmig4 & $3.677,76$ & $3.681,63$ & $3.685,43$ & 0 & 0,005837 \\
Csna3 & $3.976,50$ & $3.949,86$ & $3.987,52$ & 0 & 0 \\
Ebtp4 & $1.409,48$ & $1.412,84$ & $1.415,64$ & 0,000448 & $\mathbf{0 , 0 1 7 9 6}$ \\
Elet6 & $3.522,58$ & $3.532,29$ & $3.539,06$ & 0 & 0,000234 \\
Ibvsp & $4.165,69$ & $4.178,17$ & $4.186,31$ & 0 & 0 \\
Itau4 & $4.084,89$ & $4.065,40$ & $4.084,89$ & $\mathbf{1}$ & 0 \\
Petr4 & $3.755,75$ & $3.764,37$ & $3.767,41$ & 0 & $\mathbf{0 , 0 1 3 6 7 2}$ \\
Tcsl4 & $1.325,28$ & $1.327,02$ & $1.329,64$ & 0,003147 & $\mathbf{0 , 0 2 2 0 7 3}$ \\
Tlpp4 & $3.753,45$ & $3.763,65$ & $3.766,28$ & 0 & $\mathbf{0 , 0 2 1 8 2 1}$ \\
Tnep4 & $1.314,64$ & $1.317,41$ & $1.323,66$ & 0 & 0,000407 \\
Tnlp4 & $1.501,83$ & $1.505,29$ & $1.508,22$ & 0,00035 & $\mathbf{0 , 0 1 5 4 8 9}$ \\
Vale5 & $3.955,42$ & $3.956,39$ & $3.958,47$ & $\mathbf{0 , 0 1 3 5 1 8}$ & $\mathbf{0 , 0 4 1 3 8 9}$ \\
\hline
\end{tabular}

\section{Testing Goodness of Fit}

In this section, we test the goodness of fit. For this purpose, we use the following tests and distances:

- $\chi^{2}$ test: this test was used by Eberlein and Keller (1995) and Fajardo et al. (2001). This test is not recommendable for evaluating continuous distributions (see Press et al. (1992)).On the other hand, although the chi-square test tends to reject large samples, our tests do not report that fact (table 11).

- Kolmogorov distance:this test is more suitable than chi-square test for continuous distributions. It is expressed by:

$$
K S=\max _{x \in \mathbb{R}}\left|F_{e m p}(x)-F_{e s t}(x)\right|
$$

where $F_{e m p}$ means empirical cumulative distribution function, and $F_{e s t}$ the estimated cumulative distribution function.

- Kuiper distance: this is another distance evaluation used to test goodness of fit of continuous distributions. The main difference between Kuiper and Kolmogorov distance is that the former considers upper differences to be different from lower differences, whereas in the latter all distances are considered equally. It is expressed by:

$$
K P=\max _{x \in \mathbb{R}}\left\{F_{e m p}(x)-F_{e s t}(x)\right\}+\max _{x \in \mathbb{R}}\left\{F_{e s t}(x)-F_{e m p}(x)\right\}
$$


- Anderson \& Darling distance: The main difference between this distance and Kolmogorov distance is that the former pays more attention to tail distances Hurst et al. (1995).

$$
A D=\max _{x \in \mathbb{R}} \frac{\left|F_{e m p}(x)-F_{e s t}(x)\right|}{\sqrt{F_{e s t}(x)\left(1-F_{e s t}(x)\right)}}
$$

Now we present the obtained results:

\subsection{Chi-square test}

From Table 6, we observe that with a significance level of $5 \%$ we can not reject the null hypothesis of GHD behavior for 12 assets. In the case of NIG, we can not reject the null hypothesis in 11 assets (see Table 12), and in the case of the Hyperbolic subclass, we can not reject the null hypothesis in 9 assets (see Table 11).

\section{Table 6}

$\chi^{2}$-test for the GHD

\begin{tabular}{l|c|c|c}
\hline Sample & Statistic & $P$-Value & $\mathrm{DF}^{*}$ \\
\hline Bbas4 & 23.6516 & 0.0877 & 15 \\
Bbdc4 & 34.1268 & 0.0009 & 14 \\
Brdt4 & 66.2152 & 0 & 21 \\
Cmig4 & 21.2875 & 0.1650 & 15 \\
Csna3 & 141.5970 & 0 & 19 \\
Ebtp4 & 13.6279 & 0.3410 & 11 \\
Elet6 & 21.3830 & 0.2681 & 17 \\
Ibvsp & 13.5203 & 0.5110 & 13 \\
Itau4 & 32.5035 & 0.0819 & 22 \\
Petr4 & 15.3088 & 0.7189 & 18 \\
Tcs14 & 18.9641 & 0.1630 & 13 \\
Tlpp4 & 22.5389 & 0.0841 & 14 \\
Tnep4 & 13.3699 & 0.5229 & 13 \\
Tnlp4 & 16.5175 & 0.2258 & 12 \\
Vale5 & 16.1775 & 0.4626 & 15 \\
\hline *Degrees of freedom
\end{tabular}

\subsection{Kolmogorov distance}

In Table 7 we present the Kolmogorov distances of the NIG, Hyperbolic and $\mathrm{GH}$ distributions. In the case of $\mathrm{GH}$, all samples but CSNA3 can not be rejected using $1 \%$ of significance. Ibovespa index had a $p$ value of $99.69 \%$. 
Table 7

Kolmogorov distances

\begin{tabular}{l|c|c|c|c|c|c|c}
\hline \multirow{2}{*}{ Sample } & Normal & \multicolumn{2}{|c|}{ Hyperbolic } & \multicolumn{2}{c|}{ NIG } & \multicolumn{2}{c}{ GH } \\
& $K S$ & $K S$ & $P$-Value & $K S$ & $P$-Value & $K S$ & $P$-Value \\
\hline Bbas4 & 0.0585 & 0.0202 & 0.4446 & 0.0252 & 0.1938 & 0.0236 & 0.2611 \\
Bbdc4 & 0.0682 & 0.0279 & 0.1112 & 0.0282 & 0.1052 & 0.0279 & 0.1112 \\
Brdt4 & 0.0505 & 0.0240 & 0.2380 & 0.0303 & 0.0664 & 0.0252 & 0.1914 \\
Cmig4 & 0.0559 & 0.0238 & 0.2440 & 0.0256 & 0.1779 & 0.0270 & 0.1354 \\
Csna3 & 0.0744 & 0.0355 & 0.0192 & 0.0382 & 0.0092 & 0.0501 & 0.0002 \\
Ebtp4 & 0.0699 & 0.0253 & 0.6818 & 0.0259 & 0.6537 & 0.0234 & 0.7694 \\
Elet6 & 0.0598 & 0.0150 & 0.7968 & 0.0123 & 0.9415 & 0.0103 & 0.9897 \\
Ibvsp & 0.0661 & 0.0208 & 0.3967 & 0.0166 & 0.6833 & 0.0093 & 0.9970 \\
Itau4 & 0.0681 & 0.0347 & 0.0233 & 0.0340 & 0.0276 & 0.0347 & 0.0233 \\
Petr4 & 0.0640 & 0.0142 & 0.8526 & 0.0133 & 0.8993 & 0.0126 & 0.9294 \\
Tcsl4 & 0.0458 & 0.0220 & 0.8307 & 0.0236 & 0.7594 & 0.0253 & 0.6823 \\
Tlpp4 & 0.0784 & 0.0193 & 0.4924 & 0.0225 & 0.3062 & 0.0233 & 0.2691 \\
Tnep4 & 0.0584 & 0.0187 & 0.9405 & 0.0239 & 0.7456 & 0.0219 & 0.8342 \\
Tnlp4 & 0.0597 & 0.0178 & 0.9615 & 0.0188 & 0.9387 & 0.0178 & 0.9616 \\
Vale5 & 0.0751 & 0.0099 & 0.9931 & 0.0121 & 0.9497 & 0.0108 & 0.9813 \\
\hline
\end{tabular}

\subsection{Kuiper distance}

In Table 8, we have the Kuiper distances of the Hyperbolic subclass, NIG and GH distributions. In the case of the Hyperbolic subclass, we verify that 13 samples can not be rejected using $1 \%$ of significance. In the case of NIG, we can not reject the null hypothesis for 12 samples ( $1 \%$ of significance). This test rejects only two samples at $1 \%$ in the case of $\mathrm{GH}$, but even in the rejected samples the distances evaluated in the estimates above are smaller than normal distances.

Table 8

Kuiper distances

\begin{tabular}{l|c|c|c|c|c|c|c}
\hline \multirow{2}{*}{ Sample } & Normal & \multicolumn{2}{|c|}{ Hyperbolic } & \multicolumn{2}{c|}{ NIG } & \multicolumn{2}{c}{ GH } \\
& $K P$ & $K P$ & $P$-Value & $K P$ & $P$-Value & $K P$ & $P$-Value \\
\hline Bbas4 & 0.1133 & 0.0332 & 0.2495 & 0.0391 & 0.0742 & 0.0370 & 0.1187 \\
Bbdc4 & 0.1299 & 0.0462 & 0.0109 & 0.0496 & 0.0038 & 0.0462 & 0.0109 \\
Brdt4 & 0.0969 & 0.0413 & 0.0419 & 0.0450 & 0.0152 & 0.0414 & 0.0406 \\
Cmig4 & 0.1022 & 0.0352 & 0.1662 & 0.0392 & 0.0694 & 0.0419 & 0.0356 \\
Csna3 & 0.1299 & 0.0677 & 0 & 0.0754 & 0 & 0.1000 & 0 \\
Ebtp4 & 0.1259 & 0.0442 & 0.4531 & 0.0452 & 0.4124 & 0.0393 & 0.6615 \\
Elet6 & 0.1190 & 0.0290 & 0.4651 & 0.0228 & 0.8434 & 0.0188 & 0.9754 \\
Ibvsp & 0.1306 & 0.0280 & 0.5278 & 0.0253 & 0.6975 & 0.0172 & 0.9924 \\
Itau4 & 0.1164 & 0.0470 & 0.0086 & 0.0554 & 0.0005 & 0.0470 & 0.0086 \\
Petr4 & 0.1225 & 0.0254 & 0.6948 & 0.0259 & 0.6648 & 0.0226 & 0.8574 \\
Tcsl4 & 0.0839 & 0.0418 & 0.5533 & 0.0431 & 0.4980 & 0.0424 & 0.5260 \\
Tlpp4 & 0.1549 & 0.0351 & 0.1668 & 0.0363 & 0.1309 & 0.0349 & 0.1748 \\
Tnep4 & 0.1101 & 0.0364 & 0.7757 & 0.0448 & 0.4274 & 0.0412 & 0.5768 \\
Tnlp4 & 0.1177 & 0.0349 & 0.8357 & 0.0375 & 0.7370 & 0.0336 & 0.8761 \\
Vale5 & 0.1332 & 0.0191 & 0.9701 & 0.0236 & 0.8020 & 0.0186 & 0.9782 \\
\hline
\end{tabular}




\subsection{Anderson \& Darling distance}

The results are shown in Table 9 . We observe that this distance clearly shows the difference of fitness in the tail distributions. By analyzing the distances in comparison with the Hyperbolic distribution, we can deduce that the NIG is better as far as tail fitness is concerned. This test shows that GHD fit better tails than the Hyperbolic distribution. However, it provides similar results to the ones obtained using NIG.

Table 9

Anderson \& Darling distances

\begin{tabular}{l|c|c|c|c}
\hline Sample & Normal & Hyperbolic & NIG & GH \\
\hline Bbas4 & 137028000 & 3.1496 & 0.8093 & 1.1909 \\
Bbdc4 & 51579.5 & 0.1288 & 0.1681 & 0.1288 \\
Brdt4 & 485.583 & 0.2186 & 0.1473 & 0.2428 \\
Cmig4 & 10296 & 0.4513 & 0.2218 & 0.0720 \\
Csna3 & 7.1407 & 0.0710 & 0.0764 & 0.1546 \\
Ebtp4 & 118781 & 2.2745 & 0.5098 & 0.0762 \\
Elet6 & 51495.5 & 0.4730 & 0.1832 & 0.0837 \\
Ibvsp & 72825.7 & 2.6809 & 0.3718 & 0.0831 \\
Itau4 & 4.6648 & 0.0755 & 0.0680 & 0.0755 \\
Petr4 & 67.0476 & 0.1674 & 0.0720 & 0.0405 \\
Tcs14 & 1849990 & 2.8609 & 1.0892 & 0.2211 \\
Tlpp4 & 51523.5 & 0.5175 & 0.3044 & 0.0579 \\
Tnep4 & 305.017 & 0.7696 & 0.2051 & 0.1743 \\
Tnlp4 & 119529 & 5.9456 & 1.2154 & 0.1753 \\
Vale5 & 51523.5 & 4.7077 & 0.7483 & 0.3998 \\
\hline
\end{tabular}

\section{Derivative Pricing}

Since Black and Scholes (1973), closed formulas for European calls have been studied, but these models assume that the underlying distribution of the logreturns is normal. More recently, Prause (1999) and Raible (2000) presented the Lévy Generalized Hyperbolic process, where they assume that the log-returns of assets follow a GHD or one of its subclasses. Motivated by the good fit shown by the GHD in relation to Brazilian assets, we price European calls on Brazilian assets assuming that their log-returns follow a GHD, then, we compare with the price obtained by the Black and Scholes formula.

\subsection{Convolution of generalized hyperbolic distributions}

The first step in derivative pricing is to calculate the GHD convolution, except for NIG subclass, which has the closed convolution property, i.e. convolution of NIG is a NIG, as we can see in the following equation:

$$
n i g^{* t}(x ; \alpha, \beta, \delta, \mu)=n i g(x ; \alpha, \beta, t \delta, t \mu)
$$


To solve the convolution problem using other subclasses, we use Fourier transforms. More specifically, the characteristic function is obtained using a Fourier transform and a transformed function multiplication is similar to the original function convolution, so we take the following steps:

1. Apply Fourier transform in estimated GHD density.

2. Multiply this transform by as many convolutions as we need.

3. Apply the Inverse Fourier transform to obtain the GHD with t-fold convolution.

To simplify calculations we use a symmetric and centered distribution $(\beta, \mu=0)$ to guarantee that the functions are real Press et al. (1992). Then, we follow Prause (1999) and find a GHD as a function of a centered and symmetric GHD. This function is given by:

$$
G H^{* t}(x ; \alpha, \beta, \delta, \mu, \lambda)=\frac{e^{\beta x}}{M_{0}^{t}(\beta)} g h^{* t}(x-\mu t ; \lambda, \alpha, 0, \delta, 0)
$$

where $M_{0}^{t}(\beta)$ represents the moment generating function for the symmetric distribution, powered to $t$. Then we apply the Fourier transform in centered and symmetric GHD, obtaining (9). Then, we should apply the inverse Fourier transform, but it doesn't have an analytical solution.

$$
G H^{* t}(x ; \alpha, 0, \delta, 0, \lambda)=\frac{1}{\pi} \int_{0}^{\infty} \cos (u x) \varphi(u ; \alpha, \delta \lambda)^{t} d u
$$

To solve this kind of problem we use the Cooley and Tukey (1965) algorithm called Fast Fourier Transformation (FFT). We refer to Brigham (1988) and Press et al. (1992) for details on this algorithm applications. The FFT calculates the Fourier transform and the inverse Fourier transform in an efficient way. The main concern here is related to variable transformations from frequency to time domain. ${ }^{3}$ After FFT application we have the density of symmetric and centered $t$-fold convolution. To get the desired density we use (8).

\subsection{Option pricing using Esscher transforms}

To price options with underlying assets following diffusions driven by Lévy processes we need to find an equivalent martingale measure. Esscher (1932) presented

\footnotetext{
${ }^{3}$ For more details about this variable transformation and a Matlab example we refer to Hanselman and Littlefield (2001).
} 
a transform that was used by Gerber and Shiu (1994) for derivative pricing. In the case of GHD this transformation to risk-neutral world is given by:

$$
G H^{* t, \vartheta}(x ; \alpha, \beta, \delta, \mu, \lambda)=\frac{e^{\vartheta x}}{M^{t}(\vartheta)} G H^{* t}(x ; \alpha, \beta, \delta, \mu, \lambda)
$$

To find the $\vartheta$ parameter we have to solve

$$
r=\log \frac{M(\vartheta+1)}{M(\vartheta)}
$$

Where $r$ is the risk free interest rate in the same period of estimated data and $M$ is the moment generating function. The solution to this equation is obtained through numerical optimization. The last step is to obtain the European call prices. In this step we follow Keller (1997).

$$
C_{G H}=S_{0} \int_{\log \frac{K}{S_{0}}}^{\infty} G H^{* t, \vartheta+1}(x) d x-e^{-r t} K \int_{\log \frac{K}{S_{0}}}^{\infty} G H^{* t, \vartheta}(x) d x
$$

where $K$ is the strike price and $S_{0}$ is the stock price. In this case the Put-Call parity is valid:

$$
P_{G H}=C_{G H}-S_{0}+e^{-r t} K
$$

Figure 3 shows the Vale do Rio Doce call behavior when certain parameters are changed. As expected, the major sensitivity of GHD call prices occurs when the option is at the money. Then we compare GHD call prices and Black and Scholes (1973) call prices of this asset. We obtain Figure 4, which contains the difference between the prices. We can clearly see the desired W-Shape. The same analysis can be done using the Hyperbolic and NIG subclasses. 


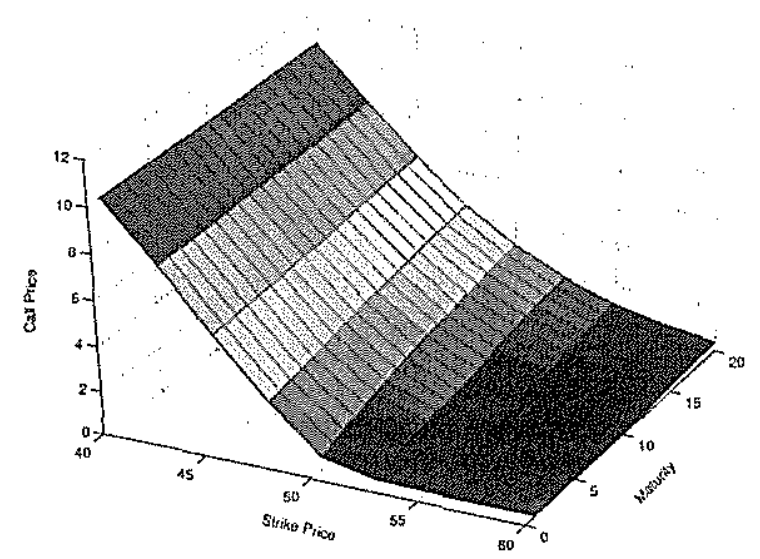

Figure 3

Vale do Rio Doce call price with $S_{0}=50$ and risk free interest rate $19 \%$ using Generalized Hyperbolic distribution

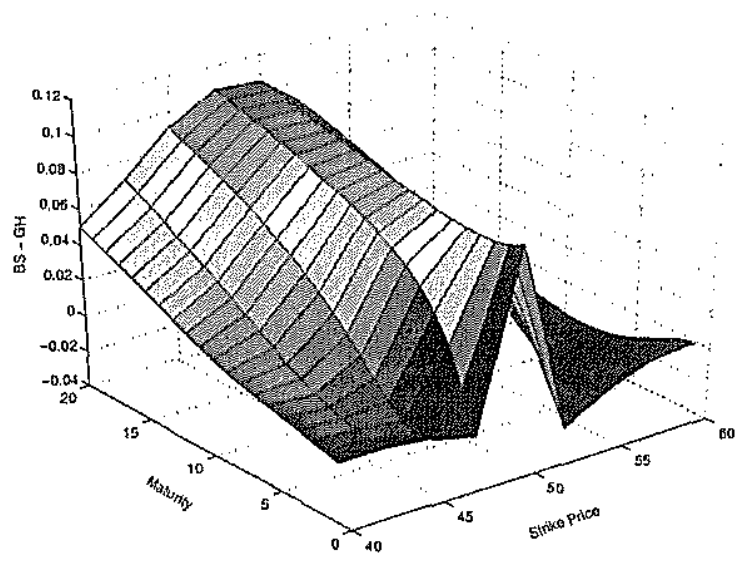

Figure 4

Black and Scholes minus GHD Vale do Rio Doce call prices for various maturities and strike prices

\section{Value at Risk}

The Value At Risk represents the worst loss, given a time period and a probability under normal market conditions Jorion (1997). In this section we briefly 
explore the parametric VaR using Normal and GHD as asset log-return distributions. In Figure 5 we have the VaR graphs for different probability levels, and we can see that the GHD get closer to empirical probability. Another technique for testing the efficiency of VaR models is Back Testing Jorion (1997). In our case,we considered a portfolio with one asset only (Vale do Rio Doce) with an initial portfolio value of $R \$ 1,00$. The initial sample used consisted of 252 observations, starting on $07 / 01 / 1994$, reaching 1590 out of sample tests. The VaR for 1 trading day holding period with $1 \%$ of probability was calculated each day. If the real loss were bigger than predicted we considered this one an exception Then we aggregated this observation and repeated the steps with another day. The results of the test are in Table 10, which brings the number of exceptions and the Kupiec (1995) test $p$-value whose null hypothesis is "The two probabilities are equal".

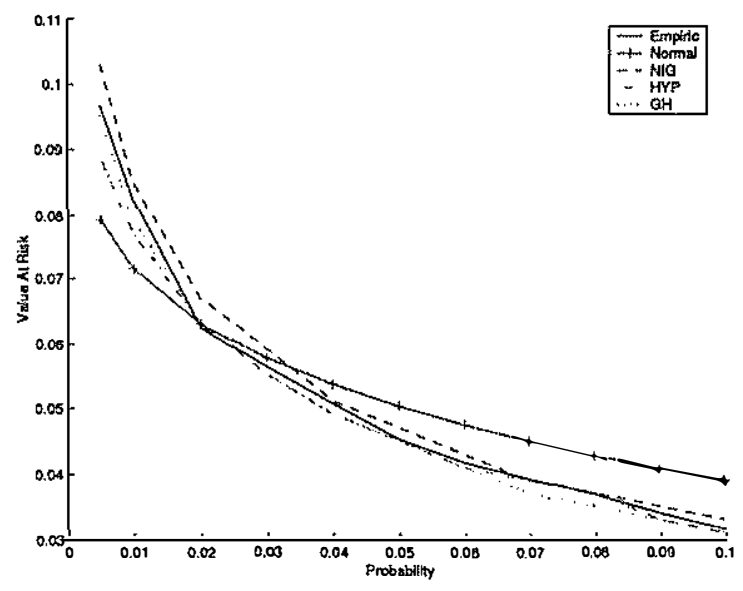

Figure 5

Value At Risk of portfolio consisting of Vale do Rio Doce assets for different probabilities with 1 trading day holding period and the portfolio value of $R \$ 1,00$

Table 10

Back-Test Exceptions and Kupiec-test $p$-value

\begin{tabular}{l|c|c|c}
\hline Distribution & Exceptions & Probability & $P$-Value \\
\hline Normal & 21 & 0.0132 & 0.22 \\
Hyperbolic & 17 & 0.0107 & 0.78 \\
N.I.G. & 16 & 0.0101 & 0.98 \\
G.H. & 16 & 0.0101 & 0.98 \\
\hline
\end{tabular}

The $1 \%$ of 1590 observations is 15.9 , Then, we can not have more than 16 exceptions. Therefore, the GH distributions pass the Kuipec's test and can be 
considered to estimate VaR. This evaluation method has as major criticism the fact that it measures exceptions but does not measure the size of error.

Table 11

Hyperbolic $\chi^{2}$ tests

\begin{tabular}{l|c|c|c}
\hline Sample & Statistic & $P$-Value & DF \\
\hline Bbas4 & 23.4443 & 0.0930 & 15 \\
Bbdc4 & 34.1268 & 0.0009 & 14 \\
Brdt4 & 70.7405 & 0 & 21 \\
Cmig4 & 21.3939 & 0.1607 & 15 \\
Csna3 & 55.3857 & 0 & 22 \\
Ebtp4 & 13.9941 & 0.3941 & 12 \\
Elet6 & 34.1289 & 0.0063 & 17 \\
Ibvsp & 34.4265 & 0.0008 & 14 \\
Itau4 & 32.5035 & 0.0819 & 22 \\
Petr4 & 25.8397 & 0.1695 & 19 \\
Tcs14 & 20.6518 & 0.1432 & 14 \\
Tlpp4 & 34.9870 & 0.0006 & 14 \\
Tnep4 & 16.6171 & 0.2872 & 13 \\
Tnlp4 & 19.5150 & 0.1405 & 13 \\
Vale5 & 12.2598 & 0.6807 & 14 \\
\hline
\end{tabular}

Table 12

NIG $\chi^{2}$ tests

\begin{tabular}{l|c|c|c}
\hline Sample & Statistic & $P$-Value & DF \\
\hline Bbas4 & 24.3353 & 0.0718 & 15 \\
Bbdc4 & 30.8658 & 0.0040 & 14 \\
Brdt4 & 80.2756 & 0 & 21 \\
Cmig4 & 21.2789 & 0.1654 & 15 \\
Csna3 & 92.3705 & 0 & 22 \\
Ebtp4 & 14.3936 & 0.3642 & 12 \\
Elet6 & 26.8696 & 0.0715 & 17 \\
Ibvsp & 23.5482 & 0.0617 & 14 \\
Itau4 & 56.8740 & 0 & 21 \\
Petr4 & 18.5013 & 0.5757 & 19 \\
Tcsl4 & 20.2143 & 0.1605 & 14 \\
Tlpp4 & 21.0947 & 0.1271 & 14 \\
Tnep4 & 19.8779 & 0.1269 & 13 \\
Tnlp4 & 18.0578 & 0.2055 & 13 \\
Vale5 & 16.9344 & 0.3372 & 14 \\
\hline
\end{tabular}




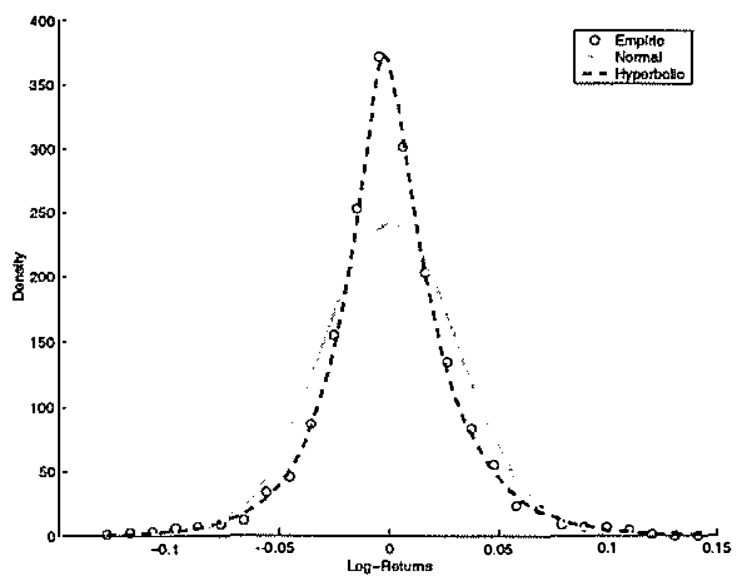

Figure 6

Vale do Rio Doce probability distribuition: empiric $\mathrm{x}$ hyperbolic $\mathrm{x}$ normal

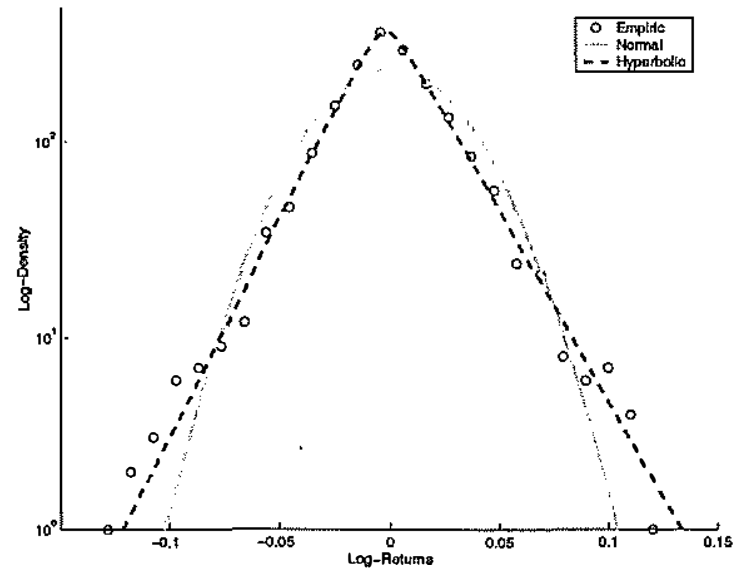

Figure 7

Vale Log-probability distribuition: empiric $\mathrm{x}$ hyperbolic $\mathrm{x}$ normal 


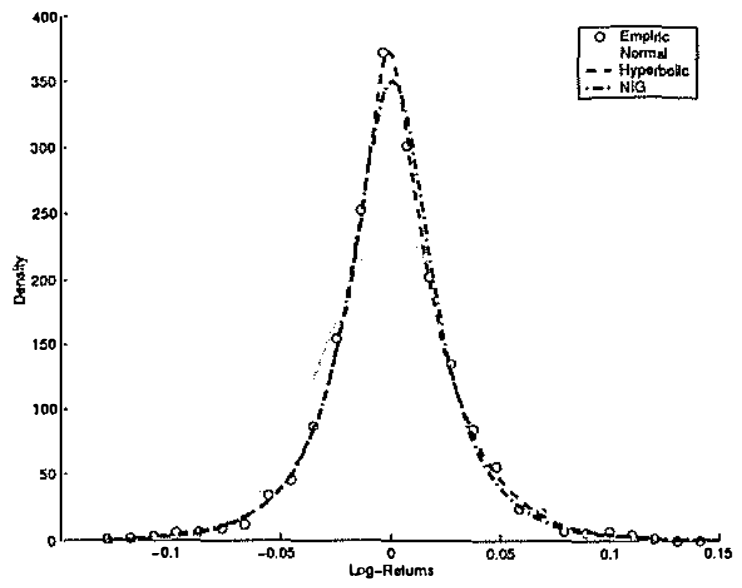

Figure 8

Vale do Rio Doce probability distribuition: empiric $\mathrm{x}$ hyperbolic $\mathrm{x}$ normal $\mathrm{x}$ NIG

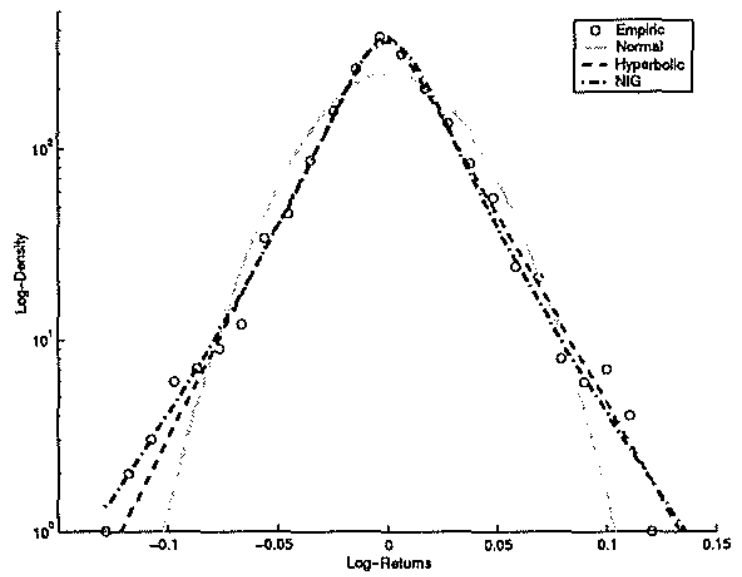

Figure 9

Vale do Rio Doce Log-probability distribuition: empiric $\mathrm{x}$ hyperbolic $\mathrm{x}$ normal $\mathrm{x}$ NIG 


\section{Conclusions}

In this paper we evaluated the goodness of fit of Generalized Hyperbolic Distributions to Brazilian asset returns and showed that they present a very good fit. Then assuming this distribution for asset returns and using Fast Fourier Transformation and Esscher (1932) transforms we priced European options. We show that it is possible to compensate a part of Black and Scholes (1973) mispricing using GHD. In the last part we calculated VaR measures and showed that GHD can be used to compute VaR.

The main limitations of the model were the computational effort needed for parameter estimation and derivative pricing plus the utilization of numerical calculus, which requires attention to precision determination. It is important to observe the trade-off between the use of a subclass or the Generalized family. The use of the Hyperbolic subclass provides faster parameter estimation and the NIG distributions simplify the derivative pricing problem (since it is closed under convolution).

An important extension of the model is to consider dependent Increments, a fact that can be captured using a NIG-S\&ARCH model as in Jensen and Lunde (2001).

\section{References}

Abramowitz, M. \& Stegun, I. A. (1968). Handbook of Mathematical Functions. Dover Publ., New York.

Baillie, R. T., Bollerslev, T., \& Mikkelsen, H. O. (1996). Fractionally integrated generalized autoregressive conditional heteroskedasticity. Journal of Econometrics, 74:3-30.

Barndorff-Nielsen, O. (1977). Exponentially decreasing distributions for the logarithm of particle size. Proceedings of the Royal Society London A, 353:401-419.

Barndorff-Nielsen, O. (1978). Hyperbolic distributions and distributions on hyperbolae. Scandinavian Journal of Statistics, 5:151-157.

Barndorff-Nielsen, O. (1997). Normal inverse Gaussian distributions and stochastic volatility modelling. Scandinavian Journal of Statistics, 24:1-13.

Barndorff-Nielsen, O. (1998). Processes of normal inverse Gaussian type. Finance and Stochastics, 2:41-68.

Barndorff-Nielsen, O. E. \& Blaesild, P. (1981). Hyperbolic distributions and ramifications: Contributions to theory and application. In Taillie, C. \& Patil, G. \& Baldessari, B., editors, Statistical Distributions in Scientific Work, volume 4, pages 19-44. Dordrecht: Reidel. 
Black, F. \& Scholes, M. (1973). The pricing of options and corporate liabilities. Journal of Political Economy, 81(3):637-654.

Blæsild, P. \& Sørensen, M. (1992). Hyp a computer program for analyzing data by means of the hyperbolic distribution. Aarhus University Research Report, 248. Department of Theoretical Statistics.

Brigham, E. O. (1988). The fast Fourier transform and its applications. Prentice Hall, New Jersey.

Cooley, J. \& Tukey, J. (1965). An algorithm for the machine calculation of complex Fourier series. Mathematics of Computations, 19(90):297-301.

Eberlein, E. (2000). Mastering risk. Prentice Hall.

Eberlein, E. \& Keller, U. (1995). Hyperbolic distributions in finance. Bernoulli, $1: 281-299$.

Esscher, F. (1932). On the probability function in the collective theory of risk. Skandinavisk Aktuarietidskrift, 15:175-195.

Fajardo, J., Schuschny, A., \& Silva, A. (2001). Lévy processes and the Brazilian market. Brazilian Review of Econometrics, 21(2):263-289.

Gerber, H. U. \& Shiu, E. S. W. (1994). Option pricing by Esscher-transforms. Transactions of the Society of Actuaries, 46:99-191.

Hanselman, D. C. \& Littlefield, B. (2001). Mastering Matlab 6 - A comprehensive tutorial and reference. Prentice Hall.

Hurst, S. R., Platen, E., \& Rachev, S. T. (1995). Option pricing for asset returns driven by subordinated processes. Working Paper, The Australian National University.

Issler, J. V. (1999). Estimating and forecasting the volatility of Brazilian finance series using ARCH models. Brazilian Review of Econometrics, 19(1):5-56.

Jensen, M. B. \& Lunde, A. (2001). The NIG-S\&ARCH model: A fat tailed, stochastic, and autoregressive conditional heteroskedastic volatility model. Aarhus University Working Paper, 83. Centre for Analytical Finance.

Jorion, P. (1997). Value at risk: The new benchmark for controlling market risk. McGraw-Hill.

Keller, U. (1997). Realistic modelling of financial derivatives. University of Freiburg, Ph.D. Thesis. 
Kupiec, P. H. (1995). Techniques for verifying the accuracy of risk measurement models. Journal of Derivatives, 3(2):73-84.

Mandelbrot, B. (1963). The variation of certain speculative prices. Journal of Business, 36:394-419.

Mazuchelli, J. \& Migon, H. S. (1999). Modelos GARCH Bayesianos: Métodos aproximados e aplicaçōes. Brazilian Review of Econometrics, 19(1):111-138.

Mendes, B. V. M. \& Duarte Jr., A. M. (1999). Robust estimation for ARCH models. Brazilian Review of Econometrics, 19(1):139-180.

Nelder, J. \& Mead, R. (1965). A simplex method for function minimization. Computer Journal, 7:308-313.

Pereira, P. L. V., Hotta, L. K., Souza, L. A. R., \& Almeida, N. M. C. G. (1999). Alternative models to extract asset volatility: A comparative study. Brazilian Review of Econometrics, 19(1):57-109.

Prause, K. (1999). The generalized hyperbolic model: Estimation, financial derivatives, and risk measures. University of Freiburg, Ph.D. Thesis.

Press, W., Teukolsky, S., Vetterling, W., \& Flannery, B. (1992). Numerical recipes in C. Cambridge University Press, Cambridge.

Raible, S. (2000). Lévy processes in finance: Theory, numerics, and empirical facts. University of Freiburg, Ph.D. Thesis.

Rydberg, T. (1997). Why financial data are interesting to statisticians. Aarhus University Working Paper, 5. Centre for Analytical Finance. 
